\title{
The effect of glutamine on Dehydroepiandrosterone-induced polycystic ovary syndrome rats
}

\author{
Gengxiang $\mathrm{Wu}^{1,2^{*}}$, Xue $\mathrm{Hu}^{3}$, Jinli Ding ${ }^{1,2}$ and Jing Yang ${ }^{1,2}$
}

\begin{abstract}
Background: Previous studies have shown that chronic inflammation and oxidative stress may play an important role in the pathophysiology of polycystic ovary syndrome (PCOS), and glutamine (GIn) have showed the antiinflammatory and antioxidant properties. So the aim of this study is to investigate the effect of glutamine supplementation on PCOS rats.

Methods: Female Sprague-Dawley rats were randomly assigned into four groups ( $n=10$ /group), control group, PCOS group, PCOS $+0.5 \mathrm{~g} / \mathrm{kg}$ Gln group and PCOS+ $1.0 \mathrm{~g} / \mathrm{kg}$ Gln group. All the PCOS rats were administrated with $6 \mathrm{mg} / 100 \mathrm{~g}$ dehydroepiandrosterone (DHEA) for 20 consecutive days, all the PCOS+Gln groups were intraperitoneal injected glutamine twice in the next morning after the last DHEA injection. All the samples were collected $12 \mathrm{~h}$ after the last administration. Ovarian histological examinations were analyzed and the concentration of serum hormone, inflammatory and oxidative stress factors were measured.

Results: There was no obvious ovarian histological change among the PCOS group and PCOS+Gln groups. All the detected inflammation factors [C-reactive protein, interleukin (IL)-6, IL-18, tumor necrosis factor] showed significantly higher in all the PCOS groups compared to the control group $(P<0.01)$, and were significantly decreased with the supplementation of $0.5 \mathrm{~g} / \mathrm{kg}$ glutamine $(P<0.01)$. Concentrations of superoxide dismutase were significantly lower in all the PCOS groups $(P<0.01)$ compared to the control group, and increased significantly with the supplementation of $0.5 \mathrm{~g} / \mathrm{kg}$ glutamine $(P<0.01)$. Serum concentrations of malondialdehyde, nitric oxide synthase and nitric oxide were significantly higher in PCOS group $(P<0.01)$ compared with the control group, and significantly decreased to the comparative levels of control group with supplementation of $0.5 \mathrm{~g} / \mathrm{kg}$ glutamine $(P<0.01)$.
\end{abstract}

Conclusion: There is low-grade inflammation and oxidative stress in DHEA-induced PCOS rats. The supplementation of $0.5 \mathrm{~g} / \mathrm{kg}$ glutamine could effectively ameliorate the inflammation and oxidative stress conditions of PCOS.

Keywords: Polycystic ovary syndrome, Dehydroepiandrosterone, Glutamine, Inflammatory factor, Oxidative stress

\footnotetext{
* Correspondence: xpwugengxiang@126.com

${ }^{1}$ Reproductive Medical Centre, Renmin Hospital of Wuhan University, Wuhan 430060, Hubei Province, People's Republic of China

${ }^{2}$ Hubei Clinical Research Center for Assisted Reproductive Technology and Embryonic Development, Wuhan 430060, People's Republic of China

Full list of author information is available at the end of the article
}

(c) The Author(s). 2020 Open Access This article is licensed under a Creative Commons Attribution 4.0 International License, which permits use, sharing, adaptation, distribution and reproduction in any medium or format, as long as you give appropriate credit to the original author(s) and the source, provide a link to the Creative Commons licence, and indicate if changes were made. The images or other third party material in this article are included in the article's Creative Commons licence, unless indicated otherwise in a credit line to the material. If material is not included in the article's Creative Commons licence and your intended use is not permitted by statutory regulation or exceeds the permitted use, you will need to obtain permission directly from the copyright holder. To view a copy of this licence, visit http://creativecommons.org/licenses/by/4.0/. The Creative Commons Public Domain Dedication waiver (http://creativecommons.org/publicdomain/zero/1.0/) applies to the data made available in this article, unless otherwise stated in a credit line to the data. 


\section{Introduction}

Polycystic ovary syndrome (PCOS) is one of the most common endocrine diseases in women of reproductive age, with a prevalence ranging from 9 to 18\% [1]. This syndrome is characterized by ovulatory dysfunction, polycystic ovarian morphology, insulin resistance and hyperandrogenism [2]. However, the pathogenesis is still unknown.

Previous studies have shown that chronic inflammation and oxidative stress may play an important role on the pathophysiology of PCOS [2-4]. Chronic inflammation is characterized by an increasing in proinflammatory cytokines and chemokines which is associated with insulin resistance [5]. There are researches have revealed that serum oxidative markers are significantly increased in patients with PCOS compared with the normal [6]. Therefore, supplementation with compounds containing anti-inflammatory and antioxidant properties may be effective in improving the pathological condition in PCOS.

Recently, extensive scientific attention has been paid on glutamine (Gln), which is the most abundant amino acid in the body, including plasma and skeletal muscles, and has anti-inflammatory and antioxidant effects as well as modulating the heat-shock protein response during stress $[7,8]$. Moreover, glutamine levels will be decreased under stress, which will activate inflammatory processes, raising the rate of protein degradation [8].

Considering the anti-inflammatory and antioxidant properties of glutamine, the present study aimed at investigating the effect of glutamine supplementation on PCOS rats.

\section{Materials and methods}

\section{Animals and treatments}

Forty female Sprague-Dawley (SD) rats (aged 23 days) weighing $80-90 \mathrm{~g}$ were obtained from the Laboratory Animal Centre of Wuhan University (Wuhan, China). The rats were maintained in groups in a well-ventilated room at $22 \pm 2{ }^{\circ} \mathrm{C}$, on a $12 \mathrm{~h}$ light $/ 12 \mathrm{~h}$ dark cycle with free access to food and water. The rats were randomly assigned into four groups ( $n=10$ /group), control group, PCOS group, PCOS+ $0.5 \mathrm{~g} / \mathrm{kg}$ Gln group and PCOS +1.0 $\mathrm{g} / \mathrm{kg}$ Gln group. The PCOS rat model was established according to our previous study [9]. Briefly, all the PCOS rats were daily injected (subcutaneously) with $6 \mathrm{mg} / 100$ $\mathrm{g}$ dehydroepiandrosterone (DHEA) (Aladdin Reagent Co., Ltd., Shanghai, China) which was dissolved in olive oil for 20 consecutive days, the control group was injected with an equivalent olive oil for 20 consecutive days. Glutamine (Double-Crane Pharmaceutical Co., Ltd., Beijing, China) was administered by intraperitoneal injection in the next morning after the last DHEA injection, the second injection of glutamine was administered $4 \mathrm{~h}$ later, PCOS group and control group were given the same amount of saline. The investigation was conducted in accordance with NIH guidelines (NIH Pub. No.85-23, revised 1996) and was approved by Animal Care and Use Committee of the Wuhan University.

\section{Sample prep2aration}

Twelve hours after the last administration, all the rats were anesthetized with isoflurane on the morning fasting state. The ovarian were fixed in $4 \%$ paraformaldehyde in $0.1 \mathrm{M}$ phosphate buffer ( $\mathrm{pH}$ 7.4) for 6-8 $\mathrm{h}$, dehydrated in the ascending series of ethanol, clarified in xylene and embedded in paraffin. Blood was collected through cardiac puncture into non-heparinized sample tube and centrifuged at $2318 \times \mathrm{g}$ for $20 \mathrm{~min}$ at room temperature and the serum was stored in $-80{ }^{\circ} \mathrm{C}$ until required for biochemical assay. All the rats were euthanized after all the samples were collected.

\section{Histology}

The ovaries embedded in paraffin were sliced into $4 \mu \mathrm{m}$ thick sections. The sections were deparaffinized with $x y-$ lene and rehydrated with descending series of ethanol, and stained by hematoxylin and eosin (H\&E) according to standard procedures (G1005, Servicebio, Wuhan, China).

\section{Biochemical analysis}

Enzyme-linked immunosorbent assay (ELISA) kits were used to measure the serum concentrations of hormones [follicle stimulating hormone (FSH), luteinizing hormone $(\mathrm{LH})$, testosterone $(\mathrm{T})$, estradiol $(\mathrm{E} 2)$, progesterone $(\mathrm{P})$, insulin], inflammatory factors [C-reactive protein (CRP), interleukin (IL)-6, IL-18, tumor necrosis factor (TNF)$\alpha$ ], and oxidative stress factors [superoxide dismutase (SOD), malondialdehyde (MDA), nitric oxide synthase (NOS) and nitric oxide (NO)]. The assay kits obtained from Elabscience ${ }^{\bullet}$ (Wuhan, China) for $\mathrm{T}$ (E-EL-0072c), CRP (E-EL-R0022c), IL-6(E-EL-R0015c), IL-18(E-ELR0567c), TNF- $\alpha$ (E-EL-R0019c), Cusabio Biotech CO., Ltd. (Wuhan, China) for FSH (CSB-E06869r), LH (CSBE12654r), E2 (CSB-E05110r), P (CSB-E07282r), insulin (CSB-E05070r), Nanjing Jiancheng Bioengineering Institute (Nanjing, China) for SOD(A001-1), MDA(A003-1), NOS(A014-2), NO(A013-2). There were standard curves used to calculate for all these kits. The coefficients of variation within and between plates were less than $10 \%$. The fasting blood glucose (FBG) level was detected by blood glucose meter (SAFE-ACCU, Sinocare, Shenzhen, China) according to the manual. Homeostasis model assessment of insulin resistance (HOMA-IR) was calculated by (concentration of serum insulin $\times$ FBG)/22.5.

\section{Statistical analysis}

Data was analyzed using SPSS version 16.0. Mean value and SD were calculated with conventional method. 
Comparisons among groups were carried out by oneway ANOVA, multiple comparisons between groups were carried out by LSD test. A $P$ value below 0.05 was considered to be statistically significant.

\section{Results}

\section{Pathological observations}

The ovarian weight in the PCOS groups [PCOS group: $(12.1 \pm 1.2) \mathrm{mg}, \mathrm{PCOS}+0.5 \mathrm{~g} / \mathrm{kg}$ Gln group: $(12.6 \pm 1.5)$ $\mathrm{mg}$, PCOS $+1.0 \mathrm{~g} / \mathrm{kg}$ Gln group:(12.4 \pm 1.8$) \mathrm{mg}]$ showed significantly higher than the controls $[(6.6 \pm 0.5) \mathrm{mg}](P<$ $0.05)$, however, there was no significance among all the PCOS groups. The ovaries in control group showed normal ovarian morphology and there were follicles and luteal formation at different stages of development, the latter is an indicator of ovulation. The ovaries in PCOS group and PCOS+Gln groups showed a large number of cystic dilatation and fewer corpus luteums, however, there was no obvious change among the PCOS group and PCOS+Gln groups (Fig. 1).

\section{Serum hormones}

Serum concentrations of $\mathrm{T}$ and $\mathrm{LH}$ were significantly higher in all the PCOS groups (include PCOS group and PCOS+Gln groups) than the control group (Fig. 2a, c), and the concentrations of $\mathrm{T}$ were increasing with the dosage of supplementation of glutamine, but the concentrations of LH were decreasing with the dosage of supplementation of glutamine. However, the concentrations of E2, FSH and P didn't show significant difference among all the groups (Fig. 2b, d, f).

\section{Serum insulin and fasting blood glucose}

Serum concentrations of insulin were significantly higher in all the PCOS groups than the control group $(P<0.01$, Fig. 3a), and the HOMA-IR showed the same tendency
(Fig. 3c), although the fasting blood glucose (FBG) didn't show significant difference among the control and all PCOS groups $(P>0.05$, Fig. $3 \mathrm{~b})$.

\section{Serum inflammation factors}

All serum concentrations of the inflammation factors (IL-6, IL-18, TNF- $\alpha$ and CRP) detected in this study showed significantly higher in all the PCOS groups compared to the control group $(P<0.01$, Fig. 4$)$, and all these factors were significantly decreased in the lower supplementation of glutamine $(P<0.01)$.

\section{Serum oxidative stress factors}

Serum concentrations of SOD were significantly lower in PCOS groups $(P<0.01$, Fig. $5 \mathrm{a})$ compared to the control group, and increased significantly with the lower supplementation of glutamine $(P<0.01)$. Serum concentrations of MDA, NO and NOS were significantly higher in PCOS group $(P<0.01$, Fig. $5 \mathrm{~b}, \mathrm{c}, \mathrm{d})$ compared with the control group, and significantly decreased to the comparative levels of control group with the lower supplementation of glutamine $(P<0.01)$.

\section{Discussion}

PCOS is a common, clinically heterogeneous endocrine disorder, which is associated with endocrinopathy and metabolic abnormalities [10]. Ovulatory dysfunction, polycystic ovarian morphology, insulin resistance and hyperandrogenism are the typical characteristics [2]. More and more studies found that PCOS is associated with chronic inflammation and oxidative stress [2-4, 11]. Glutamine is the most abundant free amino acid in human circulation [12]. It is an important source of energy and a precursor of glutathione, and plays an essential role in several metabolic processes, such as protein and nucleic acid synthesis [13], and it also has anti-
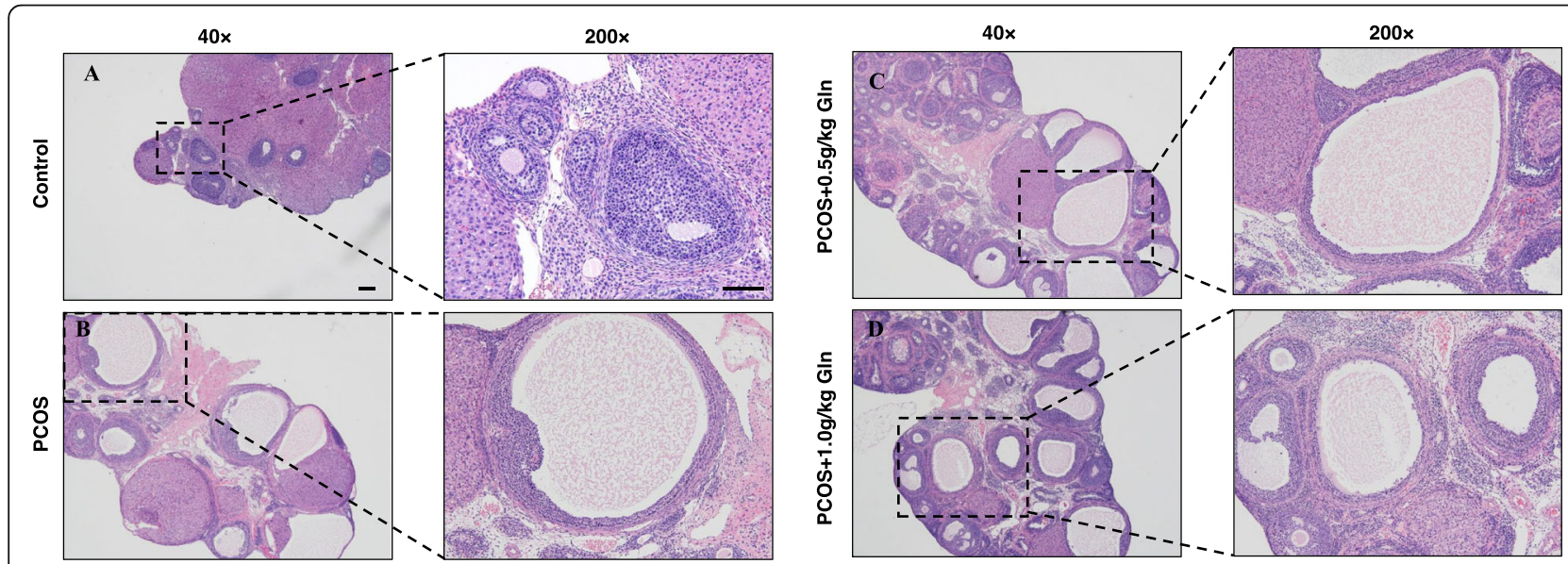

Fig. 1 Pathological images of ovarian tissues in control (a), PCOS (b)and PCOS+ $0.5 \mathrm{~kg} / \mathrm{kg}$ Gln group (c) and PCOS+ $1.0 \mathrm{~kg} / \mathrm{kg}$ Gln group (d). Hematoxylin and eosin staining, (magnification: left pictures $\times 40$, right pictures $\times 200$ ) 


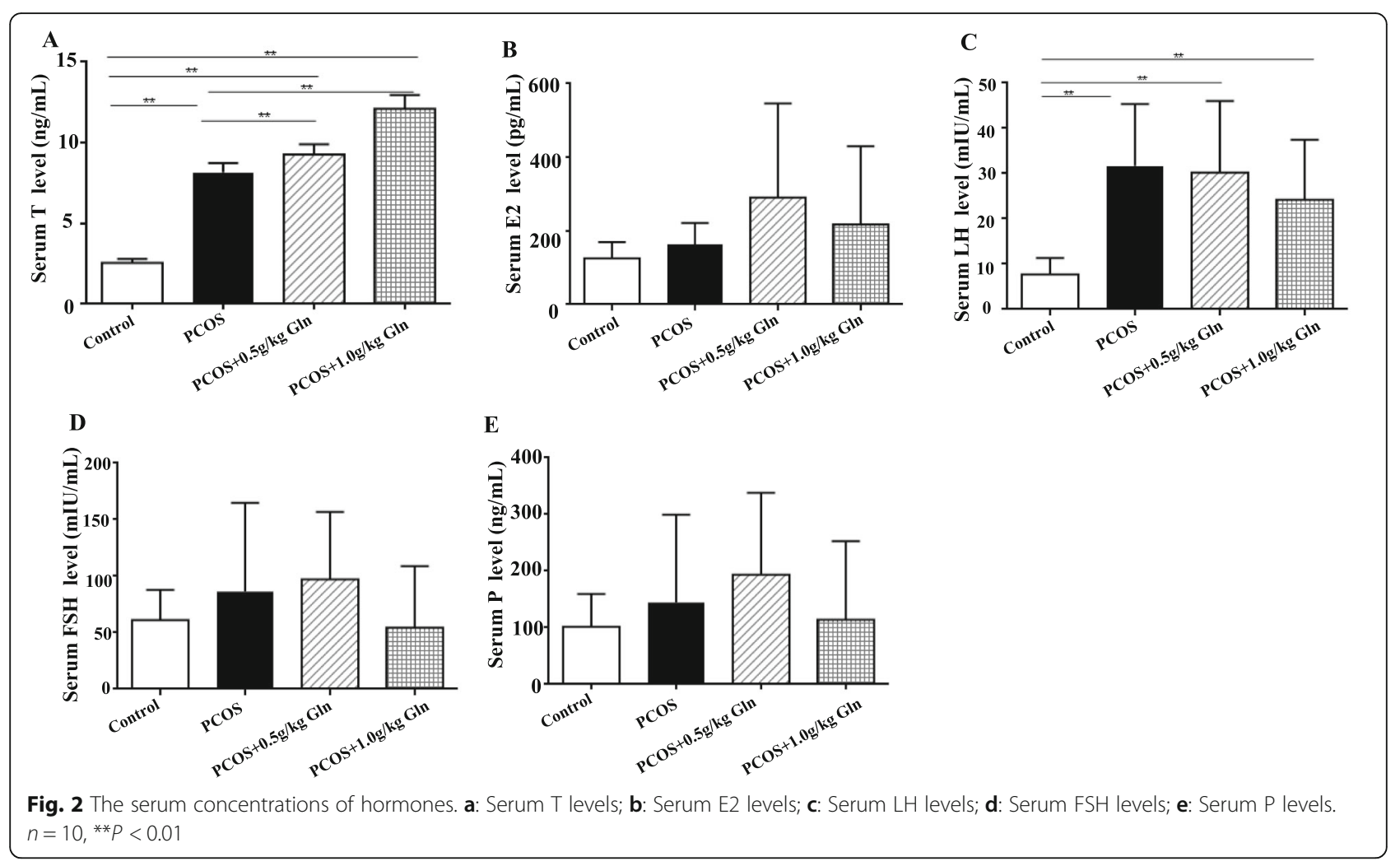

inflammatory and antioxidant effects as reported by previous studies $[7,8]$.

In the present study, the hormones and ovarian morphology changes, as well as insulin resistance in the DHEA-induced PCOS and PCOS+glutamine rats were in accordance with the characteristic of PCOS patients as previous studies $[2,9]$. Significant decrease in the level of some amino acids including glutamine was observed in plasma in PCOS patients compared with the controls, these changes reflect the various metabolic pathways that participate in physiological functions [10]. However, there are rare studies about glutamine on hormones and ovaries. Olaniyi et al. [12] found that glutamine can attenuate the decrease of the serum concentrations of FSH, $\mathrm{LH}$ and $\mathrm{T}$ in cadmium-treated male rats for a consecutive 30 days' administration. Gholipour et al. [14] reported that diet supplementing with Lglutamine for 40 days significantly increased levels of FSH and LH in guinea fowls. Our study also showed that $\mathrm{T}$ concentration was increased by the supplementation of glutamine in PCOS rats, and it increased with the dosage of the glutamine, but the LH concentration was decreased by supplementation of glutamine, even more, the concentration of FSH, E2, P and the ovaries didn't show obvious changes among these different groups. Meanwhile, the insulin resistance situation was also not
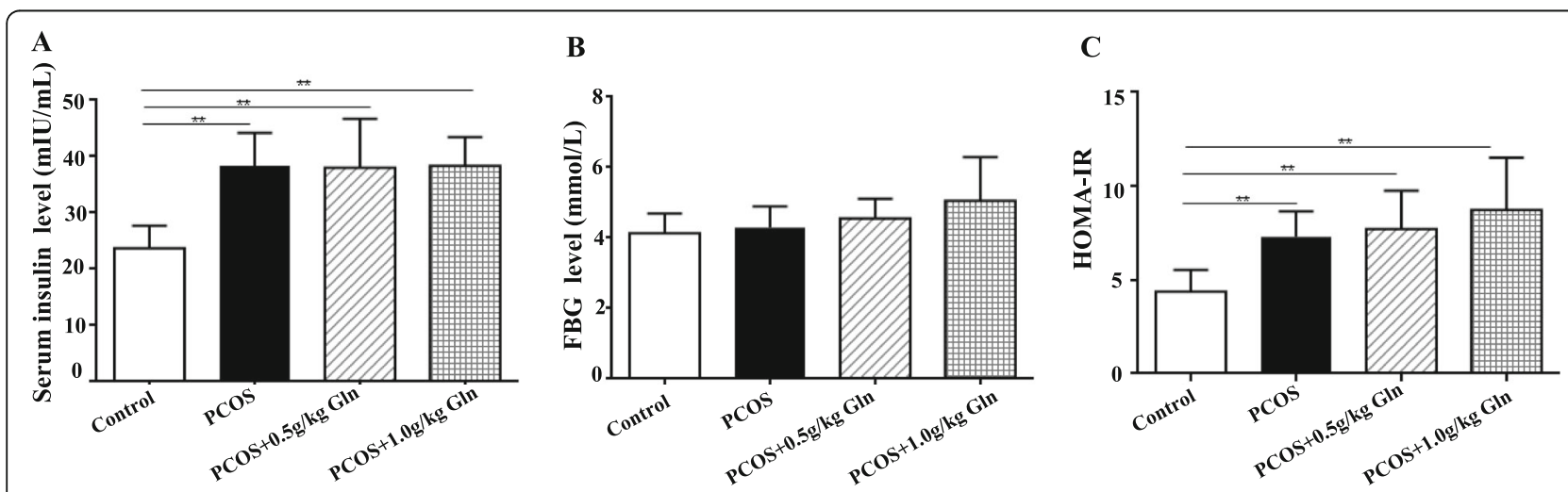

Fig. 3 The serum concentrations of insulin and fasting blood glucose (FBG). a: insulin; b: fasting blood glucose; c: HOMA-IR. $n=10,{ }^{* *} P<0.01$ 


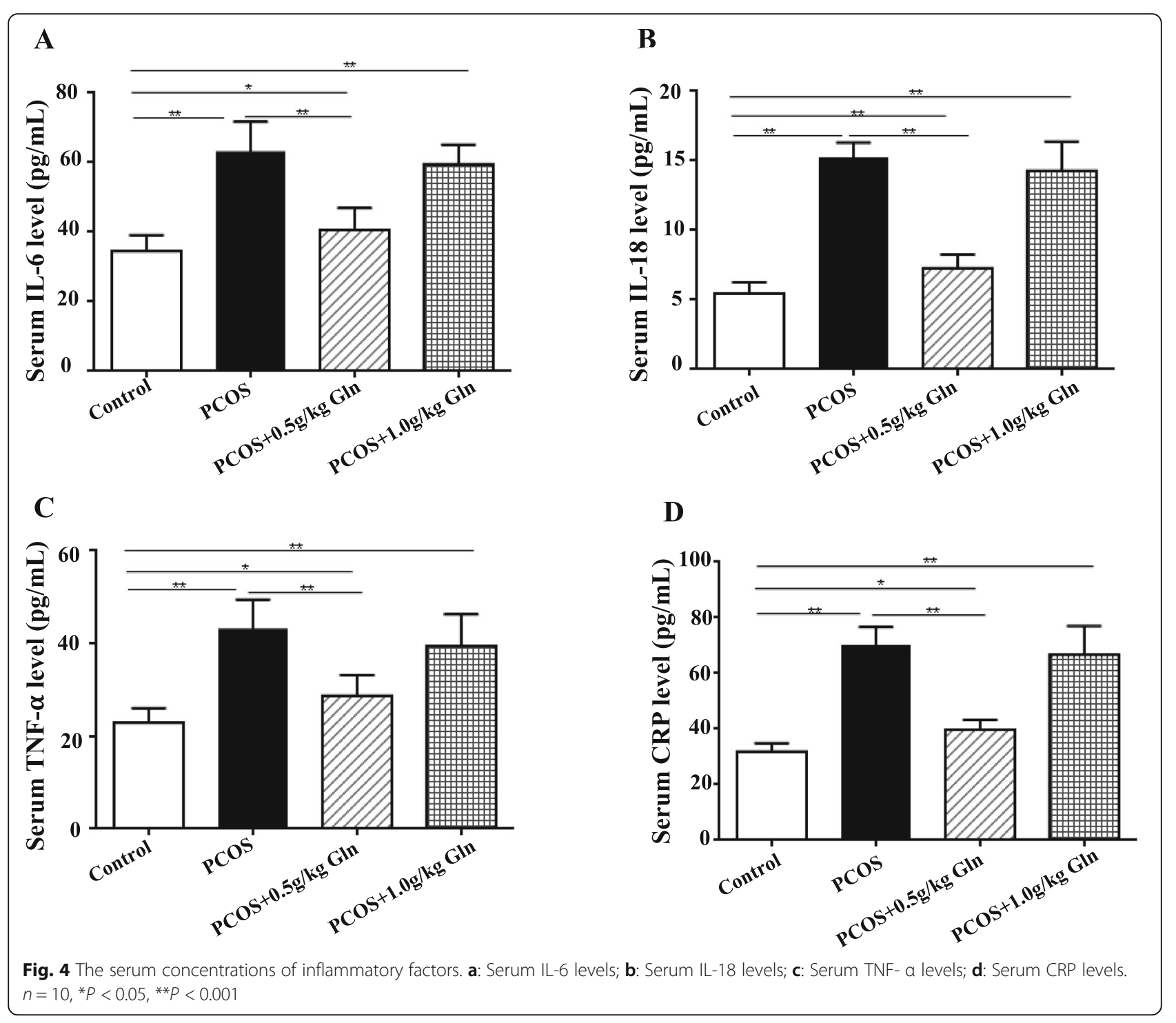

ameliorated in the PCOS groups. That may due to the short duration of glutamine administration (only twice in 1 day in this study), and it may need a longer time to recover the changes of hormones and ovarian morphology in PCOS.

Previous studies reported that there is low-grade inflammation associated with PCOS [10, 15], and our study found that serum inflammation factors levels, such as IL-6, IL-18, TNF- $\alpha$ and CRP, were significantly elevated in PCOS rats. Previous studies showed that the agents such as curcumin [15], resveratrol [16] which have anti-inflammatory effects can improve the lowgrade inflammation state by decreasing the levels of TNF- $\alpha$, IL- 6 and CRP, including the PCOS rats. Glutamine also showed an anti-inflammation effect in inflammatory diseases, the treatment with glutamine has the potential to decrease of inflammatory cytokines [17].
Glutamine can attenuate muscle inflammation, maintaining the concentration of muscle TNF- $\alpha$, IL- 6 and IL10 close to basal levels observed in the control group [8], glutamine supplementation following traumatic brain injury could inhibit NF-kB activation and downregulate pro-inflammatory cytokine expression (TNF- $\alpha$, IL-1b and IL-6) [18], and it also could decrease intestinal and plasma TNF- $\alpha$ in the induced intestinal inflammation rats [19]. Moreover, glutamine could decrease IL-6 in the intestine in human volunteers [20]. In the present study, the serum concentrations of IL-6, IL-18, TNF- $\alpha$ and CRP in PCOS rats were significantly decreased by supplementation of glutamine, especially in the $0.5 \mathrm{~g} / \mathrm{kg}$ group, which indicated glutamine could also attenuate the low-grade inflammation of PCOS.

A lot of studies have revealed that oxidative markers in circulation are significantly increased in PCOS 


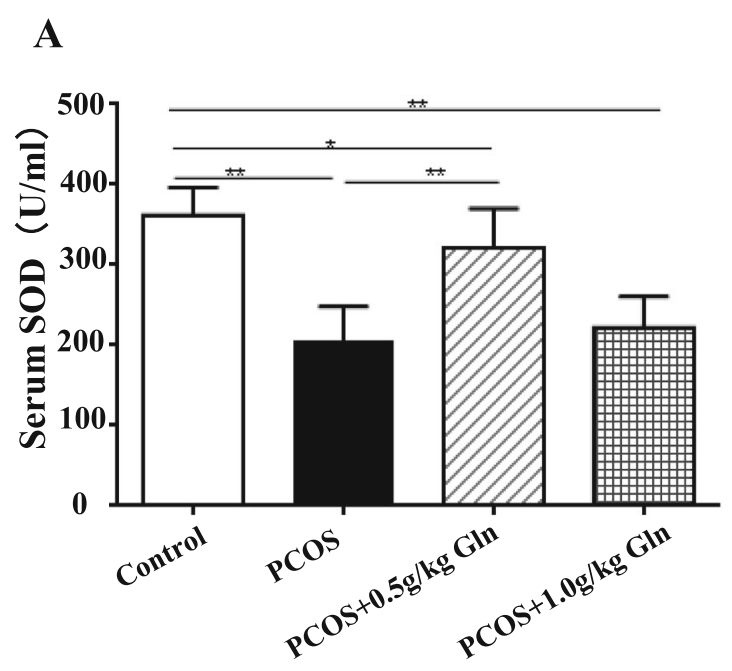

C

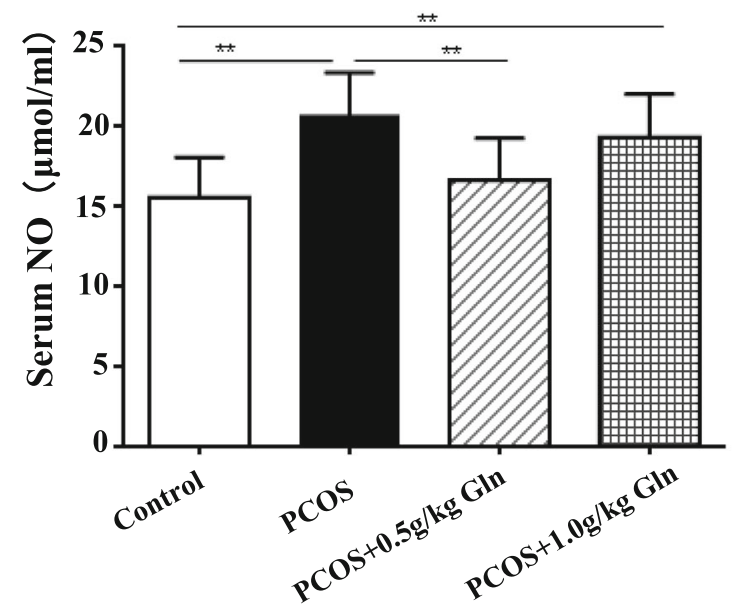

B

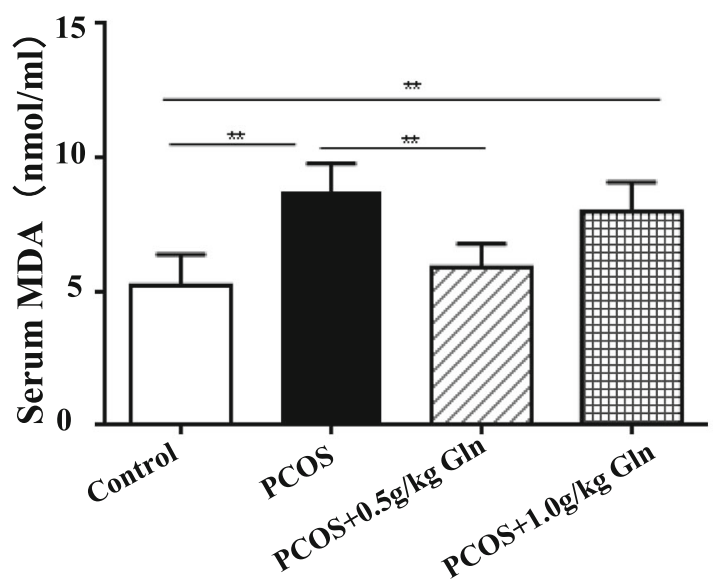

D

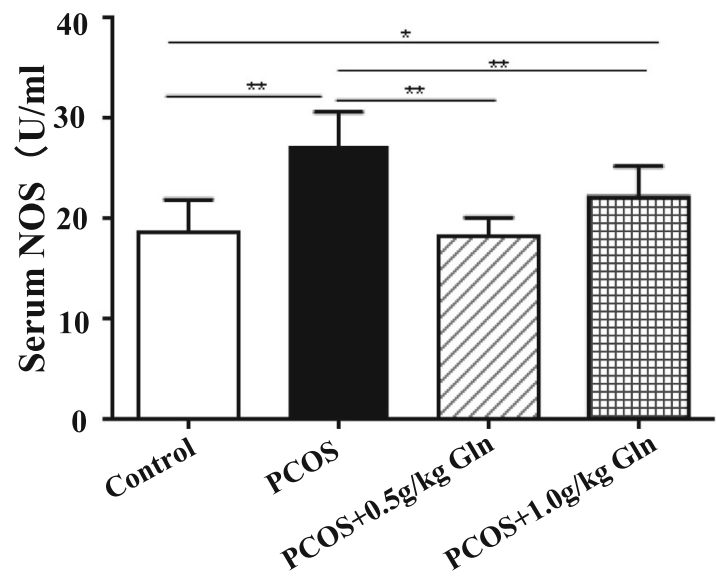

Fig. 5 The serum concentrations of oxidative stress factors. a: Serum SOD levels; b: Serum MDA levels; c: Serum NO levels; $\mathbf{d}$ : Serum NOS levels. $n=10,{ }^{*} P<0.05,{ }^{* *} P<0.001$

patients compared with the normal controls and a crucial role in the PCOS pathogenesis is played by oxidative stress $[3,4]$. Oxidative stress is considered as the imbalance between oxidants and antioxidants and the increased production of reactive oxygen species (ROS) [4]. In the present study, some serum oxidative markers were detected and the results showed that serum concentrations of SOD were significantly lower in PCOS groups, MDA, NO and NOS were significantly higher in PCOS group compared with the control group, which was consistent with previous study on PCOS patients [21]. These findings dedicated that there is an oxidative stress in these DHEA-induced PCOS rats. Furthermore, results of the present study showed that the supplementation of $0.5 \mathrm{~g} / \mathrm{kg}$ glutamine in PCOS rats could significantly increase the serum concentrations of SOD and maintain the concentrations of MDA, NO and NOS to basal levels observed in the control rats, all of these showed the antioxidant effects of glutamine. The effects of glutamine on improving antioxidant ability have been indicated in previous studies. Nemati et al. [7] reported that supplementation of glutamine can reduce oxidative stress and improve the antioxidant system of healthy adult males after an exhaustive exercise. Szpetnar et al. [22] found that glutamine is effective in reducing manganeseinduced oxidative stress by increasing glutathione peroxidase and SOD activity and decreasing in MDA level.

\section{Conclusions}

The present study demonstrates that DHEA-induced PCOS rats showed the similar hormones and ovarian morphology changes as PCOS patients, and there is lowgrade inflammation and oxidative stress in PCOS rats. The supplementation of $0.5 \mathrm{~g} / \mathrm{kg}$ glutamine could effectively ameliorate the inflammatory and oxidative stress conditions of PCOS, however, it may need a longer 
administration time of glutamine to recover the changes of hormones and ovarian morphology in PCOS.

\section{Abbreviations}

PCOS: Polycystic ovary syndrome; DHEA: Dehydro-epiandrosterone; T: Testosterone; LH: Luteinizing hormone; E2: Estradiol; FSH: Follicle stimulating hormone; P: Progesterone; CRP: C-reactive protein; IL6: Interleukin 6; IL-18: Interleukin 18; TNF- a: Tumor necrosis factor a; SOD: Superoxide dismutase; MDA: Malondialdehyde; NO: Nitric oxide: NOS: Nitric oxide synthase

\section{Acknowledgements}

Not applicable.

\section{Authors' contributions}

WGX, the first author and the corresponding author, designed the research and drafted the manuscript. HX conducted the experiments. DJL analyzed and interpreted the data and made all the figures. YJ guided the research and the writing. All authors have read and approved the final manuscript.

\section{Funding}

This work was financed by Grant-in-aid for scientific research from the National Natural Science Foundation of China (No. 81873817 and No. 81601240).

\section{Availability of data and materials}

The datasets used and analysed during the current study are available from the corresponding author on reasonable request.

\section{Ethics approval and consent to participate}

The investigation was for all animal experiments approved by Animal Care and Use Committee of the Wuhan University.

\section{Consent for publication}

Not applicable.

\section{Competing interests}

The authors declare that they have no competing interests.

\section{Author details}

${ }^{1}$ Reproductive Medical Centre, Renmin Hospital of Wuhan University, Wuhan 430060, Hubei Province, People's Republic of China. ${ }^{2}$ Hubei Clinical Research Center for Assisted Reproductive Technology and Embryonic Development, Wuhan 430060, People's Republic of China. ${ }^{3}$ Reproductive Medical Centre, Taihe Hospital, Shiyan 442000, Hubei Province, People's Republic of China.

Received: 12 February 2020 Accepted: 15 April 2020

Published online: 09 May 2020

\section{References}

1. March WA, Moore VM, Willson KJ, Phillips DI, Norman RJ, Davies MJ. The prevalence of polycystic ovary syndrome in a community sample assessed under contrasting diagnostic criteria. Hum Reprod. 2010;25(2):544-51. https://doi.org/10.1093/humrep/dep399.

2. Azziz R, Carmina E, Chen Z, Dunaif A, Laven JS, Legro RS, Lizneva D, Natterson-Horowtiz B, Teede HJ, Yildiz BO. Polycystic ovary syndrome. Nat Rev Dis Primers. 2016;2:16057. https://doi.org/10.1038/nrdp.2016.57.

3. Emidio GD, Placidi M, Rea F, Rossi G, Falone S, Cristiano L, Nottola S, D'Alessandro AM, Amicarelli F, Palmerini MG, Tatone C. MethylglyoxalDependent Glycative Stress and Deregulation of SIRT1 Functional Network in the Ovary of PCOS Mice. Cells. 2020;9(1):E209. https://doi.org/10.3390/ cells9010209.

4. Mohammadi M. Oxidative stress and polycystic ovary syndrome: a brief review. Int J Prev Med. 2019;10:86. https://doi.org/10.4103/ijpvm. IJPVM_ 576 17.

5. Ganie MA, Sahar T, Rashid A, Wani IA, Nisar S, Sathyapalan T, Vishnubhatla S, Ramakrishnan L, Parvez T, Geer I. Comparative Evaluation of Biomarkers of Inflammation Among Indian Women With Polycystic Ovary Syndrome (PCOS) Consuming Vegetarian vs. Non-vegetarian Diet. Front Endocrinol (Lausanne). 2019;10:699. https://doi.org/10.3389/fendo.2019.00699.
6. Murri M, Luque-Ramírez M, Insenser M, Ojeda-Ojeda M, Escobar-Morreale HF. Circulating markers of oxidative stress and polycystic ovary syndrome (PCOS): a systematic review and meta-analysis. Hum Reprod Update. 2013; 19(3):268-88. https://doi.org/10.1093/humupd/dms059.

7. Nemati A, Alipanah-Moghadam R, Molazadeh L, Naghizadeh BA. The effect of glutamine supplementation on oxidative stress and matrix metalloproteinase 2 and 9 after exhaustive exercise. Drug Des Devel Ther. 2019;13:4215-23. https://doi.org/10.2147/DDDT.S218606.

8. Raizel R, Leite JS, Hypólito TM, Coqueiro AY, Newsholme P, Cruzat VF, Tirapegui J. Determination of the anti-inflammatory and cytoprotective effects of I-glutamine and I-alanine, or dipeptide, supplementation in rats submitted to resistance exercise. Br J Nutr. 2016;116(3):470-9. https://doi. org/10.1017/S0007114516001999.

9. Wu G, Hu X, Ding J, Yang J. Abnormal expression of HSP70 may contribute to PCOS pathology. J Ovarian Res. 2019;12(1):74. https://doi.org/10.1186/ s13048-019-0548-7.

10. Sun L, Hu W, Liu Q, Hao Q, Sun B, Zhang Q, Mao S, Qiao J, Yan X. Metabonomics reveals plasma metabolic changes and inflammatory marker in polycystic ovary syndrome patients. J Proteome Res. 2012;11(5):2937-46. https://doi.org/10.1021/pr3000317.

11. Darabi $P$, Khazali H, Mehrabani NM. Therapeutic potentials of the natural plant flavonoid apigenin in polycystic ovary syndrome in rat model: via modulation of pro-inflammatory cytokines and antioxidant activity. Gynecol Endocrinol. 2019;30:1-6. https://doi.org/10.1080/09513590.2019.1706084.

12. Olaniyi KS, Amusa OA, Oniyide AA, Ajadi IO, Akinnagbe NT, Babatunde SS. Protective role of glutamine against cadmium-induced testicular dysfunction in Wistar rats: involvement of G6PD activity. Life Sci. 2020;242: 117250. https://doi.org/10.1016/j.lfs.2019.117250.

13. Oliveira G, Dias C, Pelosi P, Rocco P. Understanding the mechanisms of glutamine action in critically ill patients. An Acad Bras Cienc. 2010;82:417-30

14. Gholipour V, Chamani M, Aghdam Shahryar H, Sadeghi AA, Aminafshar M. Effects of dietary L-glutamine supplement on performance, egg quality, fertility and some blood biochemical parameters in Guinea fowls (Numida meleagris). Kafkas Universitesi Veteriner Fakultesi Dergisi. 2017;23(6):903-10.

15. Mohammadi S, Karimzadeh Bardei L, Hojati V, Ghorbani AG, Nabiuni M. Anti-Inflammatory Effects of Curcumin on Insulin Resistance Index, Levels of Interleukin-6, C-Reactive Protein, and Liver Histology in Polycystic Ovary Syndrome-Induced Rats. Cell J. 2017;19(3):425-33. https://doi.org/10.22074/ cellj.2017.4415.

16. Ghowsi M, Khazali H, Sisakhtnezhad S. Evaluation of TNF-a and IL-6 mRNAs expressions in visceral and subcutaneous adipose tissues of polycystic ovarian rats and effects of resveratrol. Iran J Basic Med Sci. 2018;21(2):16574. https://doi.org/10.22038/IJBMS.2017.24801.6167.

17. Jing $L$, Wu Q, Wang F. Glutamine induces heat-shock protein and protects against Escherichia coli lipopolysaccharide-induced vascular hyporeactivity in rats. Crit Care. 2007:11(2):R34

18. Chen G, Shi J, Qi M, Yin H, Hang C. Glutamine decreases intestinal nuclear factor kappa B activity and pro-inflammatory cytokine expression after traumatic brain injury in rats. Inflamm Res. 2008;57(2):57-64. https://doi.org/ 10.1007/s00011-007-7101-7.

19. Li N, Liboni K, Fang MZ, Samuelson D, Lewis P, Patel R, Neu J. Glutamine decreases lipopolysaccharide-induced intestinal inflammation in infant rats. Am J Physiol Gastrointest Liver Physiol. 2004;286(6):G914-21.

20. Coëffier M, Miralles-Barrachina O, Le Pessot F, Lalaude O, Daveau M, Lavoinne A, Lerebours E, Déchelotte P. Influence of glutamine on cytokine production by human gut in vitro. Cytokine. 2001;13(3):148-54.

21. Enechukwu Cl, Onuegbu AJ, Olisekodiaka MJ, Eleje GU, Ikechebelu Jl, Ugboaja JO, Amah UK, Okwara JE, Igwegbe AO. Oxidative stress markers and lipid profiles of patients with polycystic ovary syndrome in a Nigerian tertiary hospital. Obstet Gynecol Sci. 2019;62(5):335-43. https://doi.org/10. 5468/ogs.2019.62.5.335.

22. Szpetnar M, Luchowska-Kocot D, Boguszewska-Czubara A, Kurzepa J. The influence of manganese and glutamine intake on antioxidants and neurotransmitter amino acids levels in Rats' brain. Neurochem Res. 2016; 41(8):2129-39. https://doi.org/10.1007/s11064-016-1928-7.

\section{Publisher's Note}

Springer Nature remains neutral with regard to jurisdictional claims in published maps and institutional affiliations. 